\title{
AN ANALYTICAL CRITERION FOR THE COMPLETENESS OF RIEMANNIAN MANIFOLDS
}

\author{
WILLIAM B. GORDON
}

\begin{abstract}
If $M$ is a (not necessarily complete) riemannian manifold with metric tensor $g_{i j}$, and $f$ is any proper real valued function on $M$, then $M$ is necessarily complete with respect to the metric $\tilde{g}_{i j}=$ $g_{i j}+\left(\partial f / \partial x^{i}\right)\left(\partial f / \partial x^{j}\right)$. Using this construction one can easily prove that a riemannian manifold is complete if and only if it supports a proper function whose gradient is bounded in modulus.
\end{abstract}

1. It is known that every differentiable manifold supports a complete riemannian structure. Indeed, Nomizu and Ozeki [6] have shown that every riemannian manifold is conformally equivalent to a complete riemannian manifold. In this present note we shall describe a method for constructing complete riemannian metrics which is exceedingly simple and provides a necessary and sufficient condition for the completeness of a riemannian structure. Namely, we shall show that a riemannian manifold is complete if and only if it supports a proper function whose gradient is bounded in modulus.

We begin by recalling some basic definitions. All manifolds under discussion will be assumed to be connected paracompact Hausdorff spaces which are locally homeomorphic to open euclidean $m$-discs. A riemannian manifold $M$ of class $C^{3}$ with metric tensor $g=\left(g_{i j}\right)$ is said to be complete if either of the following two equivalent conditions hold:

(i) Every geodesic arc $\gamma=\gamma(t)$ can be extended for all values of $t$.

(ii) $M$ is complete with respect to the distance function $d(p, q)=$ (infimum of the set of arc lengths of paths joining $p$ to $q$ ).

Recall that in order for $M$ to be complete it suffices that there exist a single point $p$ such that every goedesic $\gamma=\gamma(t)$ with $\gamma(0)=p$ can be extended for all values of $t$; also, every pair of points of a complete riemannian manifold can be joined by a geodesic of minimum length. (For details and references see e.g. [4] or [5].) If $b=\left(b_{i j}\right)$ is a symmetric tensor field we write $\left(b_{i j}\right)>0$ to indicate that the form $\left(b_{i j}(p)\right)$ is positive definite at every point $p$ of $M$; i.e., that $\sum b_{i j}(p) \xi^{i} \xi^{j}>0$ for every $p$ in $M$ and nonzero vector $\xi$ in $T_{p}(M)$. By definition a riemannian metric is a symmetric tensor field $g=\left(g_{i j}\right)$ of class $C^{2}$ which is positive definite at every point.

Presented to the Society, August 28, 1972; received by the editors April 10, 1972. AMS (MOS) subject classifications (1970). Primary 53C20, 58A05; Secondary 70G05. 
Finally, we recall that a continuous map $f$ is said to be proper if $f^{-1}(K)$ is compact whenever $K$ is compact. Our main results are contained in the following two theorems:

THEOREM 1. Let $M$ be a (not necessarily complete) riemannian manifold of class $C^{3}$ with metric tensor $g=\left(g_{i j}\right)$, and let $f$ be any proper real valued function on $M$ of class $C^{3}$. Then $M$ is complete with respect to the metric $\tilde{g}=\left(\tilde{g}_{i j}\right)$ where

$$
\tilde{g}_{i j}=g_{i j}+f_{i} f_{j} \quad\left(f_{i}=\partial f / \partial x^{i}\right) .
$$

THEOREM 2. Let $M$ be as above. Then $M$ is complete with respect to $g$ if and only if there exists a proper $C^{3}$ function $f$ on $M$ such that

$$
\left(g_{i j}-f_{i} f_{j}\right)>0 \text {. }
$$

Equivalently, a riemannian manifold is complete if and only if it supports a proper $C^{3}$ function $f$ whose gradient is bounded in modulus, $(|\nabla f| \leqq$ constant $)$.

2. Proof of Theorem 1. In a few words, to prove the theorem we first observe that $\tilde{g}$ is the metric that $g$ induces on the graph of $f$, and then use the propriety of $f$ to show that its graph is Cauchy complete.

Let the product manifold $M \times R$ be endowed with the usual product metric, so that the element of arc on $M \times R$ becomes $\overline{d s^{2}}=\sum g_{i j} d x^{i} d x^{j}+$ $\overline{d z}^{2}$ where $x=\left(x^{i}, \cdots, x^{m}\right)$ represents a local coordinate chart on $M$ and $z$ a general point of $R$. The graph of $f$ will be defined to be the subspace of $M \times R$ which consists of all pairs $\{(p, f(p)) \mid p \in M\}$ and is endowed with the obvious differentiable structure which makes $G$ diffeomorphic to $M$. Hereafter we identify $G$ with $M$. Now $\tilde{g}$ is the metric which $G$ inherits from its embedding in $M \times R$, and therefore the theorem will be proved once we show that every $\tilde{g}$-Cauchy sequence in $G$ contains a $\tilde{g}$-convergent subsequence.

But if $\left\{\left(p_{n}, f\left(p_{n}\right)\right)\right\}$ is a Cauchy sequence in $G$, then $\left\{f\left(p_{n}\right)\right\}$ is a Cauchy sequence in $R$ because

$$
d_{G}\left[(p, z),\left(p^{\prime}, z^{\prime}\right)\right] \geqq d_{M \times R}\left[(p, z),\left(p^{\prime}, z^{\prime}\right)\right] \geqq\left|z-z^{\prime}\right| .
$$

Therefore by passing to a subsequence we can assume that $f\left(p_{n}\right) \rightarrow z$ for some $z$ in $R$, so that $\left\{z, f\left(p_{1}\right), f\left(p_{2}\right), \cdots\right\}$ is a compact subset of $R$. But $\left\{p_{1}, p_{2}, \cdots\right\} \subset f^{-1}\left\{z, f\left(p_{1}\right), f\left(p_{2}\right), \cdots\right\}$ and $f$ is proper. Therefore the sequence $\left\{p_{n}\right\}$ contains a convergent subsequence since it is contained in a compact set.

REMARK 1. The proof generalizes the following construction: Let $A$ be a closed subset of $R^{2}$ and let $f$ be a $C^{3}$ function defined on $R^{2}-A$. Let $G$ be the surface embedded in $R^{3}$ which lies over $R^{2}-A$ and is given by the 
relation $z=f(x, y)$. Then $G$ is complete (with respect to the induced metric) if $f$ is a proper function (on $R^{2}-A$ ).

REMARK 2. Let $g$ and $\tilde{g}$ be two metric tensors on $M$. It is a trivial matter to verify that $M$ is complete with respect to $\tilde{g}$ if $M$ is complete with respect to $g$ and $\left(\tilde{g}_{i j}-g_{i j}\right)$ is positive semidefinite at every point. Hence from (1) it follows that the graph of any function on a manifold is complete if the manifold is itself complete.

3. Proof of Theorem 2. Let $f$ be a proper $C^{3}$ function which satisfies (2). Then the assertion that $M$ is complete with respect to $g$ is an immediate consequence of Theorem 1 and the relation $g_{i j}=\left(g_{i j}-f_{i} f_{j}\right)+f_{i} f_{j}$.

We shall raise and lower indices in the usual tensorial fashion and employ the tensor summation convention. If $f$ satisfies (2), then $|\nabla f|<1$; for multiplying (2) by $f^{i} f^{j}$ and summing on repeated indices we get $|\nabla f|^{2}-$ $|\nabla f|^{4}>0$. On the other hand suppose $|\nabla f| \leqq$ constant. Multiplying $f$ by a suitably small constant we can assume that $|\nabla f|<1$. Then for any vector $\xi$ we have

$$
g_{i j} \xi^{i} \xi^{j}-\left(f_{i} \xi^{i}\right)\left(f_{j} \xi^{j}\right)=|\xi|^{2}-(\nabla f, \xi)^{2} \geqq|\xi|^{2}\left(1-|\nabla f|^{2}\right) .
$$

I.e. $\left(g_{i j}-f_{i} f_{j}\right)>0$, and we have shown that the existence of a proper function satisfying (2) is equivalent to the existence of a proper function whose gradient is bounded in modulus.

Finally, to complete the proof we have to show that every complete riemannian manifold $M$ supports a proper $C^{3}$ function $f$ whose gradient is bounded in modulus. By a well-known theorem of Nash we can assume the existence of an isometric embedding $j: M \rightarrow R^{N}$ for some $N$. Then $M$ is complete iff $M$ is a closed subspace of $R^{N}$ iff $j$ is proper. Let $w=\left(w^{1}, \cdots\right.$, $w^{N}$ ) be standard euclidean coordinates on $R^{N}$, and let $F(w)=\log \left(1+|w|^{2}\right)$. Then $F$ is a proper function on $R^{N}$ and $|\nabla F| \leqq 1$. Let $f=F \circ j=F \mid M$. Then $f$ is proper because the composition of proper maps is proper; and $|\nabla f| \leqq 1$ because at each point $p$ of $M, \nabla f(p)$ is the orthogonal projection of $\nabla F(p)$ onto $T_{p}(M)$, so that $|\nabla f(p)| \leqq|\nabla F(p)|$.

4. The theorem of Nomizu and Ozeki is an easy consequence of Theorem 2. In fact, we have the following:

COROLlaRY. Let $f$ be any proper $C^{3}$ function on $M$ and let $g=\left(g_{i j}\right)$ be any metric tensor on $M$. Then $M$ is complete with respect to the metric $\tilde{g}$ where $\tilde{g}_{i j}=e^{|\nabla f|^{2}} g_{i j}$.

Proof. Let $\nabla_{g}, \nabla_{\sim},|\cdot|_{g},|\cdot|_{\tilde{g}}$ denote the gradient operators and norms associated with $g$ and $\tilde{g}$ respectively. Then

$$
\left|\nabla_{\tilde{g}} f\right|_{\tilde{g}}^{2}=\left|\nabla_{g} f\right|_{g}^{2} \exp \left(-\left|\nabla_{g} f\right|_{g}^{2}\right) \leqq 1 / e
$$


REMARK. The existence of proper functions on $M$ is provided by Whitney's embedding theorem, which asserts that a smooth manifold $M$ can be embedded as a closed submanifold of $R^{k}$ (for $k$ sufficiently large). The existence of such an embedding being given, one can then construct a proper function on $M$ exactly as was done in the last section. Whitney's theorem also guarantees the existence of complete riemannian metrics, for as we have already mentioned in the last section, the metric induced by a closed embedding is necessarily complete.

5. We conclude by mentioning some applications to hamiltonian systems. Let $V$ be a smooth function on $M$, and consider the hamiltonian system associated with the "potential" $V$. Conditions on $V$ are known which guarantee the completeness of the hamiltonian system ([1], [2], [7]), but these conditions do not apply to the geodesic case (the case $V=0$ ) unless $M$ is compact. Moreover, these conditions often assume the completeness of the underlying riemannian manifold $M$. We now wish to show how Theorem 2 can be applied to conservative dynamical systems by means of the Jacobi metric.

Recall that the Jacobi metric $\hat{g}_{i j}$ associated with a potential $V$ is given by $\hat{g}_{i j}=(h-V) g_{i j}$, where $h$ is a constant. It is well known that every trajectory of the dynamical system with total energy $h$ can be represented as a reparametrized geodesic with respect to the Jacobi metric. Suppose for simplicity that $V \leqq 0$, so that $\hat{g}_{i j}$ is positive definite for every $h>0$. Then according to Theorem $2, \hat{g}_{i j}$ is complete if and only if there exists a proper function $f$ on $M$ such that $|\nabla f|^{2} \leqq h-V$.

Results of this kind being established, one can then apply the standard results of riemannian geometry to obtain the existence of trajectories with arbitrary given energy (within a certain range) which join two fixed points and have arbitary topological type. (Cf. [3].) Moreover, in certain situations one can use the completeness of Jacobi metrics to establish the existence of periodic trajectories. (Indeed, this seems to be the geometric content of Assumption [A] in [3].)

REMARK. There is no connection between the completeness of hamiltonian systems and the completeness of the corresponding Jacobi metric. For example, in euclidean space with the origin removed, let $V(x)=$ $-1 /|x|^{n}$ with $n \geqq 2$ (so that $V$ corresponds to a central force which is inverse cube or stronger). Then for $h>0$ the Jacobi metric is complete (but the hamiltonian system is not). Proof. In Theorem 2 set $f(x)=\log |x|$.

\section{REFERENCES}

1. D. G. Ebin, Completeness of Hamiltonian vector fields, Proc. Amer. Math. Soc. 26 (1970), 632-634. MR 43 \#4070. 
2. W. B. Gordon, On the completeness of Hamiltonian vector fields, Proc. Amer. Math. Soc. 26 (1970), 329-331. MR 43 \#2318.

3. - Physical variational principles which satisfy the Palais-Smale condition, Bull. Amer. Math. Soc. 78 (1972), 712-716.

4. R. Hermann, Differential geometry and the calculus of variations, Math. in Sci. and Engineering, vol. 49, Academic Press, New York, 1968. MR 38 \#1635.

5. S. Kobayashi and K. Nomizu, Foundations of differential geometry. Vol. 1, Interscience, New York, 1963. MR 27 \#2945.

6. K. Nomizu and H. Ozeki, The existence of complete Riemannian metrics, Proc. Amer. Math. Soc. 12 (1961), 889-891. MR 24 \#A3610.

7. A. Weinstein and J. Marsden, A comparison theorem for Hamiltonian vector fields, Proc. Amer. Math. Soc. 26 (1970), 629-631. MR 42 \#8525.

Mathematics Research Center, Naval Research laboratory, Washington, D.C. 20390 